\title{
Comparison of the Collision-Induced Dissociation of Duplex DNA at Different Collision Regimes: Evidence for a Multistep Dissociation Mechanism
}

\author{
Valérie Gabelica and Edwin De Pauw \\ Mass Spectrometry Laboratory, Department of Chemistry, University of Liege, Liege, Belgium
}

\begin{abstract}
The dissociation mechanism of duplex DNA has been investigated in detail by collisioninduced dissociation experiments at different collision regimes. MS/MS experiments were performed either in a quadrupole collision cell (hybrid quadrupole-TOF instrument) or in a quadrupole ion trap with different activation times and energies. In addition to the noncovalent dissociation of the duplex into the single strands, other covalent bond fragmentation channels were observed. Neutral base loss from the duplex is favored by slow activation. In fast activation conditions, however, the major reaction channel is the noncovalent dissociation into single strands, which is highly entropy-favored. Fast activation regimes can favor the entropy-driven noncovalent dissociation, while in slow heating conditions the competition with enthalpy-driven covalent fragmentation can completely hinder the dissociation of the complex. We also evidence that the noncovalent dissociation of DNA duplex is a multistep process involving a progressive unzipping, preferentially at terminal positions. This is proposed to be a general feature for complexes containing a high number of contributing interactions organized at the interface of the ligands. The overall (observed) dissociation kinetics of noncovalent complexes can therefore depend on a complicated mechanism for which a single transition state description of the kinetics is too simplistic. (J Am Soc Mass Spectrom 2002, 13, 91-98) (c) 2002 American Society for Mass Spectrometry
\end{abstract}

$\mathrm{T}$ The introduction of electrospray (ES) [1-4] led to tremendous development of the study of noncovalent complexes by mass spectrometry. ESI gently transfers the complexes from the solution to the gas phase by a progressive desolvation assisted by collisions in the source [5], by heating in a capillary [6], or both. Once the intact complexes have been obtained in the gas phase, they can be studied by MS/MS collision-induced dissociation (CID) in quadrupoles [7-9], in ion traps [10, 11], in ICR cells [12, 13], blackbody radiation in ICR cells $[14,15, \ldots]$, or in-source dissociation techniques (heated capillary dissociation $[16,17]$, in-source CID [13, 18-20]. All these methods give access to the relative dissociation kinetics of the complexes [21], which depends on the nature and the strength of the interactions between the constitutive units. Tandem mass spectrometry has therefore the potency to become a method of choice to probe the intrinsic interactions between biomolecules in the absence of solvent.

Currently, the interpretation of the MS/MS on large

Published online November 20, 2001

Address reprint requests to Dr. V. Gabelica, Mass Spectrometry Laboratory, Department of Chemistry, Bat. B6c, University of Liege, B-4000 Liege, Belgium. E-mail: v.gabelica@ulg.ac.be complexes is still based on a simplistic view inherited from earlier studies on small molecules. The usual picture is based on the RRKM [22-26] (Rice-Rampsberger-Kassel-Marcus) statistical theory of unimolecular dissociation. The expression of the rate constant $\mathrm{k}(\mathrm{E})$ depends on an enthalpic term (the critical energy $E_{0}$ ), and on the activation entropy (through a degeneracy factor $\sigma$ and the density of states of the reactant and the transition state). For large molecules, the link between the observed fragmentation yield and the microscopic characteristics of the complex is difficult to make because computation of the rate constant is currently impossible for systems with such a large number of degrees of freedom. Only the blackbody infrared radiative dissociation (BIRD) [27, 28] technique can give access to the Arrhenius parameters $\mathrm{E}_{\mathrm{a}}$ and $\mathrm{A}$. The interpretation of the results is still quite intuitive. The role of the energy barrier is quite easy to understand, and the fragmentation ratio is often assumed to reflect the height of the energy barrier, and therefore the strength of the noncovalent interactions. However, the role of the dissociation entropy is more difficult to visualize and is therefore somewhat neglected.

Further complication can also arise from the multiple local minima that can exist in the potential energy landscape of large molecules. The dissociation mecha- 
nism can involve multiple intermediates, and a one-step scheme may be too simplistic. Supramolecular assemblies are usually held together by multiple cooperating interactions (hydrogen bonds, Coulomb or shorter range electrostatic interactions, Van der Waals bonds, ....). Each one alone would be insufficient to stabilize the complex, but their sum can lead to a very strong complex provided that the constitutive ligands are adequately arranged in space. From a purely statistical point of view, the simultaneous breaking of all the noncovalent bonds at the same time is highly improbable, and the dissociation of noncovalent complexes involving multiple interactions is therefore more likely to proceed via a multi-step dissociation process [20].

The present article aims at highlighting some of these important aspects of the dissociation of noncovalent complexes. Except by ion mobility measurements, the intermediate steps involving incompletely dissociated complexes cannot be resolved, as the mass of the intermediate species is the same as the mass of the intact complex. We will address the problem of the multi-step dissociation of macromolecular assemblies through the competition between noncovalent dissociation of the complex and covalent fragmentation of the constitutive ligands. DNA duplexes were chosen as model compounds; these species have been quite extensively studied by source-CID and MS/MS, and strong evidence of the conservation of Watson-Crick base pairing and base stacking interactions is provided by the comparison of the dissociation rate of complexes with various sequences [14, 20, 29, 30]. Competition between the noncovalent dissociation of the complex and different covalent fragmentation reactions have been reported for DNA duplexes [14, 30] and cyclodextrin complexes with peptides $[16,31]$. The effect of the collision regime on the competition between covalent bond fragmentation and noncovalent dissociation of the complex has been investigated in detail. The present study provides evidence (1) for a multistep dissociation mechanism of duplex DNA and (2) for a highly entropyfavored noncovalent dissociation process.

\section{Experimental}

\section{Materials}

All oligonucleotides were purchased from Eurogentec (Sart-Tilman, Belgium) and used without further purification. Stock solutions of $50 \mu \mathrm{M}$ duplex were prepared by heating the single strands to $75^{\circ} \mathrm{C}$ and cooling overnight. Annealing was performed in $50 \mathrm{mM}$ aqueous ammonium acetate. A solution of $20 \mu \mathrm{M}$ duplex in 20:80 (vol/vol) methanol/50 $\mathrm{mM}$ aqueous $\mathrm{NH}_{4} \mathrm{OAc}$ was injected in the mass spectrometers.

\section{Mass spectrometry}

CID in a quadrupole collision cell. MS/MS experiments were performed on a Q-TOF2 (Micromass, Manchester,
UK) electrospray mass spectrometer, and the Z-spray source was operated in the negative ion mode at a capillary voltage of $-2350 \mathrm{~V}$. The source block temperature was $80^{\circ} \mathrm{C}$ and the desolvation gas (N2) temperature was $100^{\circ} \mathrm{C}$. The cone voltage was set to $20 \mathrm{~V}$.

CID in a quadrupole ion trap. MS/MS experiments were also performed on an LCQ (ThermoFinnigan, San Jose, CA) equipped with its standard electrospray source. The needle voltage was set to $-3.9 \mathrm{kV}$, the capillary voltage to $-20 \mathrm{~V}$ and the heated capillary temperature to $180{ }^{\circ} \mathrm{C}$. MS/MS was performed during different activation times with the advanced scan parameters of Xcalibur 1.0 software. The activation $Q$ was left at 0.250 for all experiments. The activation time can be varied from $1 \mathrm{~ms}$ to $10 \mathrm{~s}$. The longer the activation, the smaller the activation amplitude necessary to fragment the parent ion to a given extent. MS/MS experiments were carried out with activation times of $3 \mathrm{~ms}$ (the minimum to have a good quality spectrum), $30 \mathrm{~ms}$, and $300 \mathrm{~ms}$. For each activation time, the activation amplitude was varied from 15 to $30 \%, 7$ to $15 \%$, and 5 to $12 \%$ respectively. These amplitude ranges are suitable to observe the evolution from the intact duplex to its complete fragmentation.

\section{Results and Discussion}

In order to investigate the strength of noncovalent interactions in the gas phase, one usually compares the energy required to dissociate the complex (in the present case, the duplex) into its constitutive parts (the single strands). This is the noncovalent dissociation channel. The usual way to report comparative data for different complexes is to measure the appearance of the fragments upon increase of the collision energy, and to compare the energies at which $50 \%$ of the complex is dissociated. The present work does not focus on such comparisons, but is more dedicated to a discussion of both the effect of the experimental MS/MS conditions on such measurements and of the dissociation mechanism. That is why we do not report such breakdown curves, but rather show the spectra obtained in different typical activation conditions.

\section{MS/MS of 12-Mer Duplexes with Different Hydrogen Bond Content at Different Collision Regimes}

Figure 1 shows four MS/MS spectra of Duplex A (see Table 1 for the base sequence), obtained by using slower activation conditions from /a/ to /d/. CID of the duplex $^{5-}$ (noted DS) in the quadrupole collision cell of the Q-TOF2 produces the single strands (noted ss) which share the available charges. In the ion trap, the main fragmentation pathway is also the noncovalent dissociation into single strands, but another fragment appears in slow heating conditions: the duplex that has 


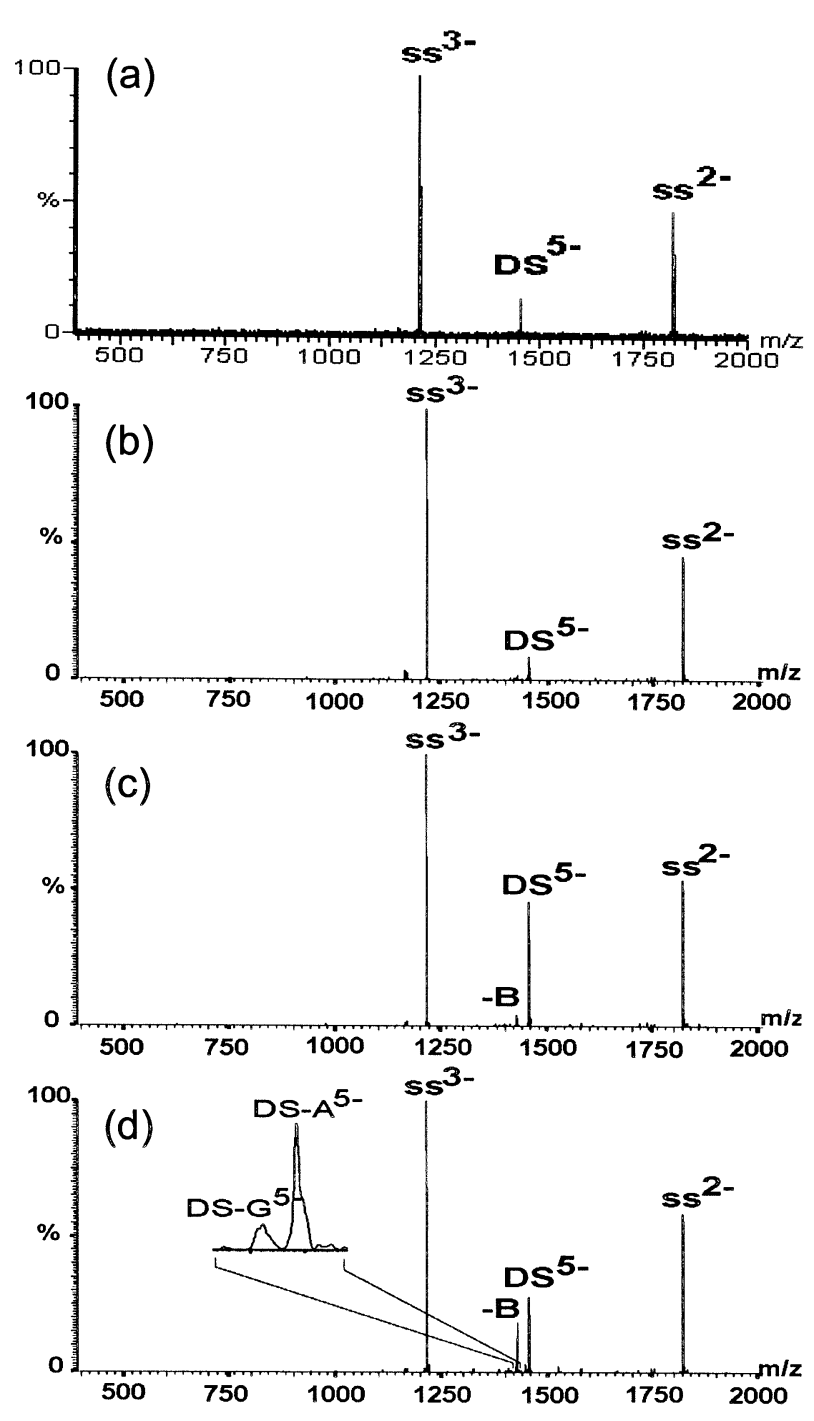

Figure 1. MS/MS spectra of Duplex A [d(CGTAAATTTACG $)_{2}{ }^{5-}$ ] recorded in different collision regimes, fastest to slowest activation from top to bottom. (a) Q-TOF2, collision energy $=16 \mathrm{eV}$. (b) LCQ, activation time $=3 \mathrm{~ms}$, activation amplitude $=22 \%$. (c) LCQ, activation time $=30 \mathrm{~ms}$, activation amplitude $=10 \%$. (d) LCQ, activation time $=300 \mathrm{~ms}$, activation amplitude $=8 \%$. The annotations are described in the text.

lost a neutral base (noted DS-B or -B), either adenine or guanine. Figure 2 shows the MS/MS spectra of Duplex B (see Table 1) recorded with the same reaction times as in Figure 1. At the highest collision energy regimes (Figure $2 \mathrm{a}$ and $\mathrm{b}$ ), the main fragmentation pathway is the noncovalent dissociation into single strands. At the lowest collision energy regimes (Figure $2 \mathrm{c}$ and $\mathrm{d}$ ), the neutral base loss from the duplex becomes more and

Table 1. Base sequences of 12-mer Duplexes A-C

\begin{tabular}{|c|c|c|}
\hline & Sequence & $\begin{array}{c}\% \text { of CG } \\
\text { base pairs }\end{array}$ \\
\hline $\bar{A}$ & $\mathrm{~d}\left(5^{\prime}-\right.$-CGTAAATTTACG-3') ${ }_{2}$ & $33 \%$ \\
\hline B & $\mathrm{d}\left(5^{\prime}-\mathrm{CGCGAATTCGCG-3')_{2 }}\right.$ & $67 \%$ \\
\hline $\mathrm{C}$ & $\mathrm{d}\left(5^{\prime}-\mathrm{CGCGGGCCCGCG-3^{ \prime } ) _ { 2 }}\right.$ & $100 \%$ \\
\hline
\end{tabular}

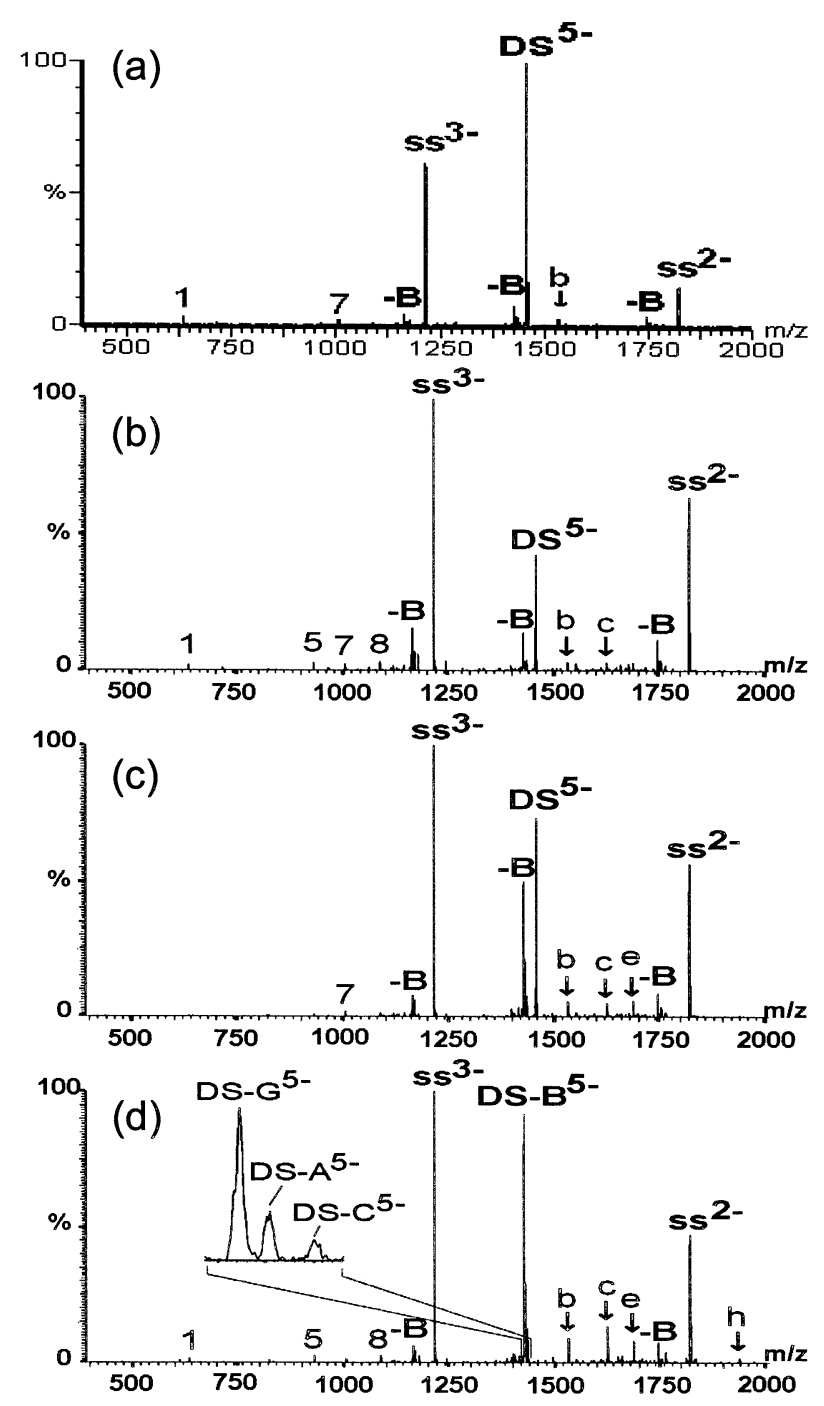

Figure 2. MS/MS spectra of Duplex B [d(CGCGAATTCGCG) ${ }_{2}{ }^{5-}$ ] recorded in different collision regimes, fastest to slowest activation from top to bottom. (a) Q-TOF2, collision energy $=22 \mathrm{eV}$. (b) LCQ, activation time $=3 \mathrm{~ms}$, activation amplitude $=24 \%$. (c) LCQ, activation time $=30 \mathrm{~ms}$, activation amplitude $=12 \%$. (d) LCQ, activation time $=300 \mathrm{~ms}$, activation amplitude $=10 \%$. The annotations are described in the text.

more important. In addition to these major peaks, the signal of other fragments appear in the spectra. Table 2 summarizes the fragments that have been observed throughout this study. Two categories of fragments can be distinguished: Type I fragments are defined as backbone fragments of single strands (noted 1, 2, 3, . .) and Type II fragments are defined as duplexes with backbone fragmentation of one of the two strands (noted $\mathbf{a}, \mathbf{b}, \mathbf{c}, \ldots$. .). The relative abundance of Type II fragments increases when slow activation conditions are used. In Figure 3, we see that the abundance of Type I and Type II fragments is even higher for Duplex C (see Table 1). Like for Duplex A and B, the neutral base loss channel is highly favored by slow activation conditions. Two bases can even be lost. Type I fragments are, however, favored when CID is performed in a quadrupole (Figure 3a). 
Table 2. Identification of the observed fragments

\begin{tabular}{rlll}
\hline \multicolumn{2}{c}{ Type I } & \multicolumn{2}{c}{ Type II } \\
\hline \hline 1 & $\mathrm{w}_{2}{ }^{-}$ & $\mathrm{a}$ & $\left(\mathrm{DS}^{-} \mathrm{a}_{5}\right)^{4-}$ \\
2 & $\mathrm{a}_{5}-\mathrm{B}^{2-}$ & $\mathrm{b}$ & $\left(\mathrm{DS}-\mathrm{a}_{4}\right)^{4-}$ \\
3 & $\mathrm{a}_{3}-\mathrm{B}^{-}$ & $\mathrm{c}$ & $\left(\mathrm{DS}^{-} \mathrm{d}_{2}-\mathrm{G}\right)^{4-}$ \\
4 & $\mathrm{a}_{6}-\mathrm{B}^{2-}$ & $\mathrm{d}$ & $\left(\mathrm{DS}-\mathrm{x}_{7}-\mathrm{G}\right)^{3-}$ \\
5 & $\mathrm{w}_{6}{ }^{2-}$ & $\mathrm{e}$ & $\left(\mathrm{DS}-\mathrm{a}_{2}\right)^{--}$ \\
6 & $\mathrm{w}_{3}{ }^{-}$ & $\mathrm{f}$ & $\left(\mathrm{DS}-\mathrm{x}_{6}-\mathrm{G}\right)^{3-}$ \\
7 & $\mathrm{a}_{4} \mathrm{~B}^{-}$ & $\mathrm{g}$ & $\left(\mathrm{DS}-\mathrm{a}_{6}\right)^{-}$ \\
8 & $\mathrm{w}_{7}{ }^{2-}$ & $\mathrm{h}$ & $\left(\mathrm{DS}-\mathrm{a}_{5}\right)^{3-}$ \\
9 & $\mathrm{w}_{8}{ }^{2-}$ & & \\
10 & $\mathrm{w}_{4}{ }^{-}$ & & \\
11 & $\mathrm{a}_{5}-\mathrm{B}^{-}$ & & \\
\hline
\end{tabular}

\section{Competition Between Noncovalent Dissociation and Covalent Fragmentation}

Besides the dissociation channel of interest corresponding to the noncovalent dissociation of the complex into its components, there can be side processes like the fragmentation of covalent bonds that, if predominant, complicate the comparison with solution-phase data, and are therefore not desirable. It has been reported that cyclodextrin-peptide complexes underwent noncovalent dissociation in CID and heated-capillary dissociation [16], so that the relative kinetic stabilities of the complexes could be determined and correlated with the binding affinities in solution. However, in BIRD experiments on the same complexes, covalent fragmentation of the peptide occurred instead, and no activation energy could be determined for the noncovalent dissociation reaction [31].

Competition between the noncovalent dissociation of the complex and different covalent fragmentation reactions have also been reported for DNA duplexes $[14,30]$. The neutral base loss is the predominant channel competing with the noncovalent dissociation. For single strands $[32,33]$ and for non-complementary duplexes [14], it has been reported that the base loss initiated the backbone fragmentation. To investigate whether the Type II fragments observed in Figures 2 and 3 are also initiated by base loss, we performed MS $^{3}$ experiments on the (DS-B) ${ }^{5-}$ species isolated from the fragmentation of Duplex B, which was isolated by a first MS/MS step in slow heating conditions (activation time $=300 \mathrm{~ms}$ ). $\mathrm{MS}^{3}$ results are displayed in Figure 4. When comparing Figures $2 b$ and $4 a$, and Figures $2 d$ and $4 \mathrm{~b}$ (same activation times for $\mathrm{DS}^{5-}$ and (DS-B) ${ }^{5-}$ respectively), we see that Type II fragments are more abundant when CID is undertaken on the (DS-B) species. Although this is not a definite proof, it suggests that (DS-B) species are the precursors of Type II fragments. Type I fragments can be produced either by fragmentation of DS-B into Type II and the complementary Type I fragments or by fragmentation of single strands that contain excess energy.

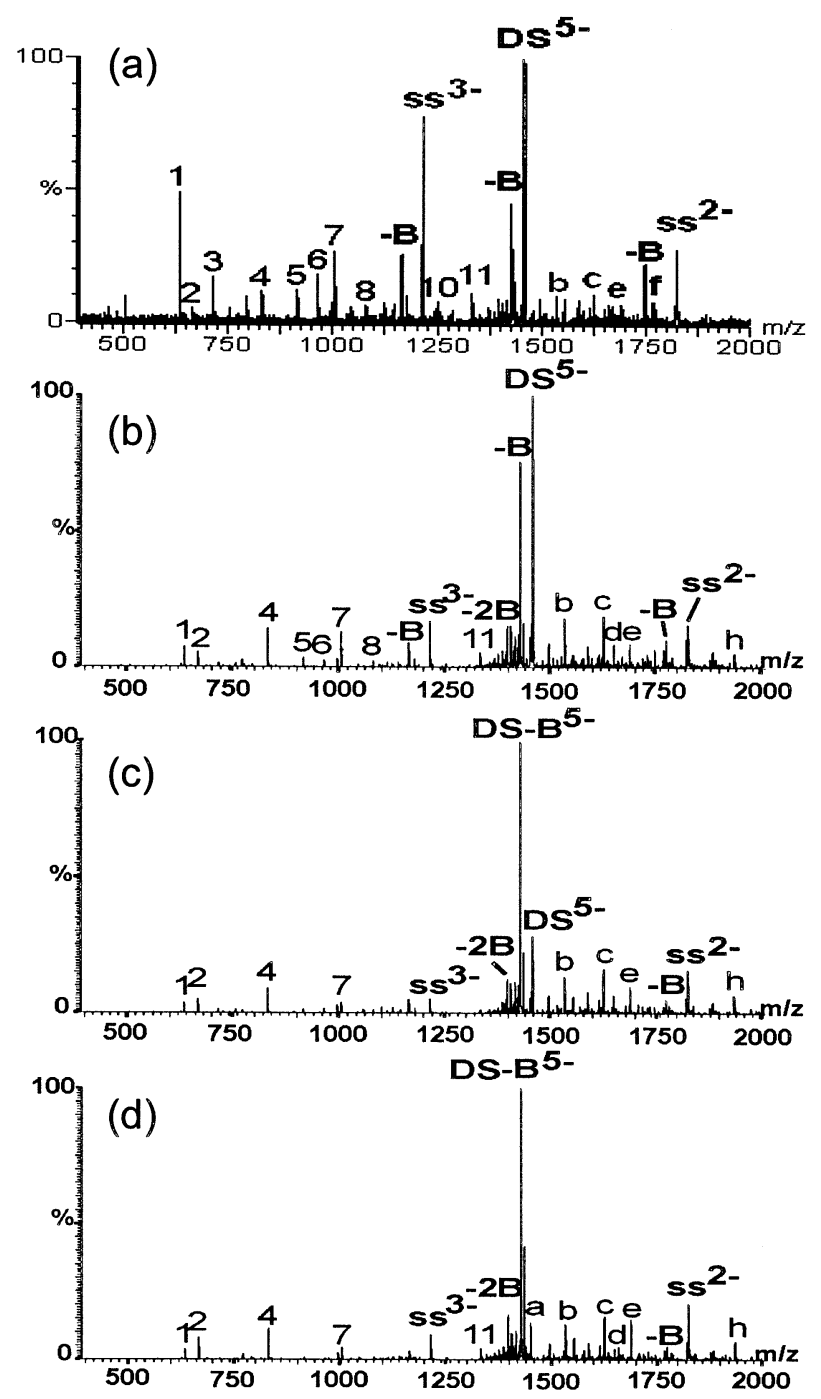

Figure 3. MS/MS spectra of Duplex C [d(CGCGGGCCCGCG) ${ }_{2}{ }^{5-}$ ] recorded in different collision regimes, fastest to slowest activation from top to bottom. (a) Q-TOF2, collision energy $=30 \mathrm{eV}$. (b) LCQ, activation time $=3 \mathrm{~ms}$, activation amplitude $=24 \%$. (c) LCQ, activation time $=30 \mathrm{~ms}$, activation amplitude $=12 \%$. (d) LCQ, activation time $=300 \mathrm{~ms}$, activation amplitude $=11 \%$. The annotations are described in the text.

\section{Influence of the Collision Regime on the Observed Reaction Channels}

Entropy-favored noncovalent dissociation. Figures 1, 2, and 3 all show that a fast activation regime favors the noncovalent dissociation channel compared to the neutral base loss, which is the major covalent fragmentation channel. Typical $k(E)$ curves reflecting the observed situation are shown in Figure 5. The fact that the relative proportion of noncovalent dissociation and neutral base loss varies with the collision regime indicates that the activation entropy of these two channels is different. Indeed, the $\mathrm{k}(\mathrm{E})$ curves can cross only if the steepness of the curves is different, and it is the activation entropy that is responsible for the steepness of the curve. As previously reported [34], the transition state 


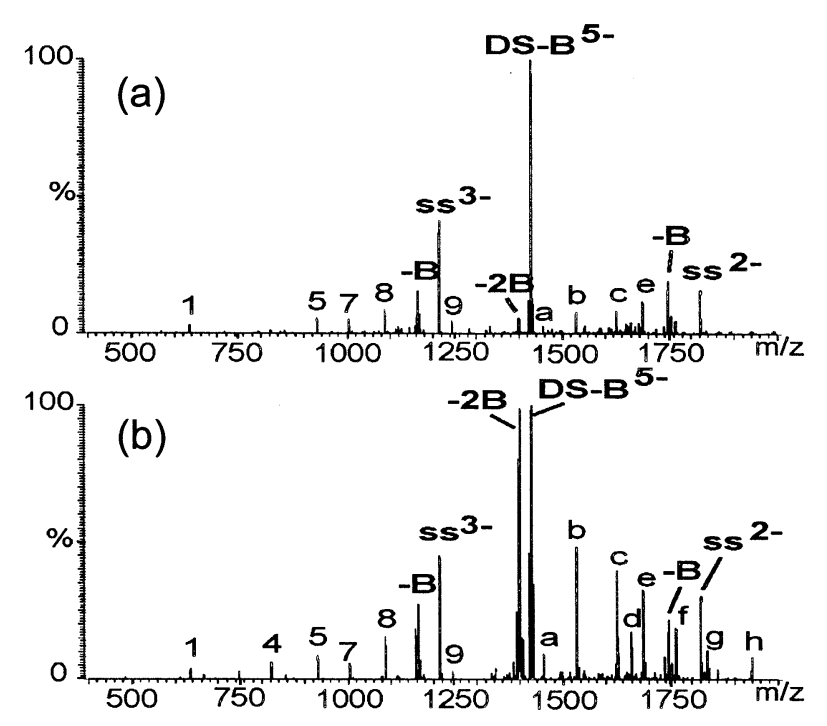

Figure 4. $\mathrm{MS}^{3}$ spectra on (DS-B) ${ }^{5-}$ isolated from Duplex B, which is produced as in Figure 2d. (a) Activation time $=3 \mathrm{~ms}$, activation amplitude $=23 \%$. (b) Activation time $=300 \mathrm{~ms}$, activation amplitude $=9 \%$. The annotations are described in the text.

for base loss is tight; it involves a complex rearrangement mechanism. This is an entropy-disfavored process: the rate constant for base loss increases less steeply with internal energy than the rate constant of an entropyfavored process. Noncovalent dissociation is, however, a highly entropy-favored process. No complex rearrangements with constrained conformations are involved. Rather, the gain in conformational freedom is enormous, especially for complexes with a large interaction interface as in the present case. The rate constant therefore rises much more steeply with internal energy.

Kinetic shift effect. The critical energy $\mathrm{E}_{0}$ for the noncovalent dissociation depends on the strength of the intermolecular interactions. The experimental threshold for the observation of the dissociation increases with the number of hydrogen bonds in the duplex $[14,20,29,30]$. The higher the number of GC base pairs (and hence the number of hydrogen bonds between the strands), the higher the collision energy necessary to reach noncovalent dissociation. For Duplex A, B, and C, the k(E) curves are assumed to be parallel, as the dissociation mechanisms are likely to be the same. When low threshold, entropy-disfavored processes are in competition with entropy-favored processes, it can happen that the respective $\mathrm{k}(\mathrm{E})$ curves cross at a given energy. In a typical energy-dependent MS/MS experiment, the reaction time is fixed, and the internal energy is increased progressively. This corresponds to a movement from left to right on a horizontal line in Figure 5. The order of appearance of the different dissociation channels depends on the order in which the $k(E)$ curves are crossed by that horizontal line. In the case of an infinitely long reaction time, the order of occurrence of the reactions when increasing the energy would depend only on the critical energy $E_{0}$, (no kinetic shift). As the time allowed for reaction diminishes, the kinetic shift effect increases: the apparent threshold is higher than the true threshold $\mathrm{E}_{0}$ and the order of appearance of the different reaction channels can change. In the LCQ, three different reaction times were studied, from 3 to $300 \mathrm{~ms}$. In such slow heating conditions, the internal energy rises by small increments, and slow processes are observed (see parts c and d in Figures 1, 2, and 3). For Duplex A, the threshold for noncovalent dissociation is low and this is the main channel observed. For Duplex B, the thresholds for base loss and noncovalent dissociation must be close, and both channels are almost equally important. For Duplex C ( $100 \%$ of GC base pairs), the rate constant for base loss is highly favored because of its lower threshold. For the latter duplex, the only way to obtain significant amounts of single strands is to perform MS/MS in a quadrupole (Figure 3a). In the Q-TOF instrument, the internal energy uptake per collision is higher than in slow heating, and the reaction time is in the order of the microsecond time scale. The kinetic shift is therefore higher than in the LCQ. This faster activation regime favors the noncovalent dissociation channels (see Figure 5). Competition between base loss and noncovalent dissociation is also observed when isolating (DS-B) $)^{5-}$ of Duplex B (Figure 4); in fast heating conditions (Figure 4a) the noncovalent dissociation of (DS-B) into the single strands (one of which with a missing base) is predominant; in slow heating conditions (Figure $4 \mathrm{~b}$ ) a second base loss occurs. Type II fragments are also favored by a slow heating of (DS$B)^{5-}$. The choice of the activation method for the

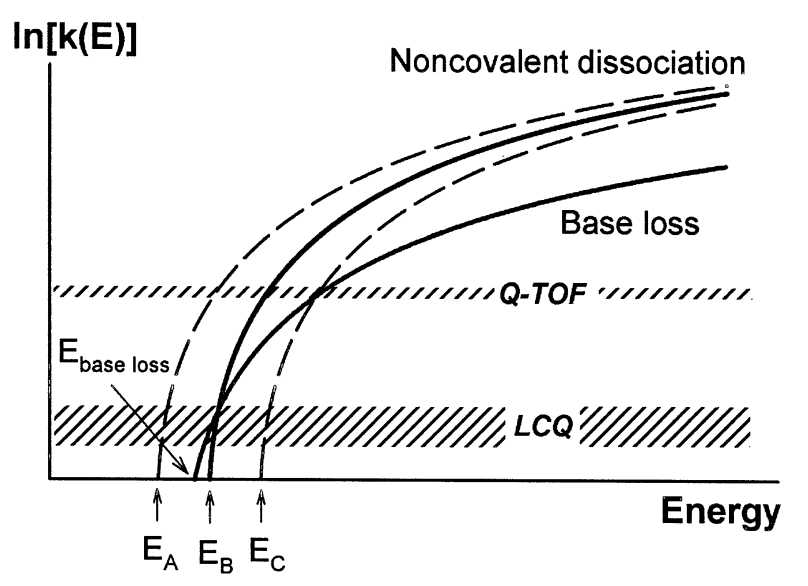

Figure 5. Schematics of the dependence of global rate constants for neutral base loss and noncovalent dissociation on the internal energy. Base loss (a rearrangement) has a low energy threshold, but the rate constant increases less steeply with the energy. Noncovalent dissociation has increasing thresholds from Duplex A to $\mathbf{C}$, and are entropy-favored processes: The rate constant increases more steeply with energy. In the LCQ (slow heating), the reaction times are varied from $3 \mathrm{~ms}$ to $300 \mathrm{~ms}$, the observed rate constants are small. In the Q-TOF2 (fast heating), the reaction time is in the microsecond range and more energy is required to obtain larger rate constants. The kinetic shift (difference between the observed threshold and the true threshold for dissociation) is larger for fast heating processes. 
Table 3. Relative intensities of adenine and guanine loss from DNA 16-mers upon slow heating conditions (LCQ, 8\%, 1s)

\begin{tabular}{lcc}
\hline Sequence & A loss* & G loss* \\
\hline \hline 5'-AAATCGCGGCGCTAAA-3' & & \\
3'-TTTAGCGCCGCGATTT-5' & $55 \%$ & $45 \%$ \\
5'-AGACTGTGAGTCAGTG-3' & & \\
3'-TCTGACACTCAGTCAC-5' & $45 \%$ & $55 \%$ \\
5'-GGGCTATAATATCGGG-3' & & \\
3'-CCCGATATTATAGCCC-5' & $33 \%$ & $67 \%$ \\
\hline
\end{tabular}

*The error on the relative intensities is $2 \%$.

MS/MS experiments is therefore critical for the study of the dissociation of the complex with no or at least minimal interference of side-reactions like covalent fragmentation.

\section{Proposed Mechanism for the Dissociation of Duplex DNA}

Terminal unzipping of the duplex. The base loss from the duplex has also driven our attention for another reason: In order for the duplex to loose a base, it must be in a partially unzipped state. The separation of the duplex into its single strands is therefore not a single-step process. From a purely statistical point of view, the one-step dissociation of the duplex is highly improbable; as discussed previously [20], the simultaneous breaking of tens of hydrogen bonds would result in a very high energy activation barrier. Rather, assuming a multi-step process with each base pair opening requiring a small activation energy, is more realistic. To investigate the preferential unzipping sites, a series of 16-mer duplexes, each containing 8 AT and 8 GC base pairs at different positions have been studied. MS/MS was performed on the duplex DS $^{6-}$ with the LCQ mass spectrometer with $8 \%$ activation amplitude during $1 \mathrm{~s}$ (slow heating conditions). The relative abundance of $(\mathrm{DS}-\mathrm{G})^{6-}$ and (DS-A) ${ }^{6-}$ have been measured. The results are summarized in Table 3. Clearly, terminal bases are more readily lost than internal base pairs. Control experiments have confirmed that for single strands there is no such dependence on the position (data not shown). Unzipping, which is essential for base loss, therefore seems to occur preferentially at terminal base pairs.

Proposed reaction mechanism. The assembling of all these pieces of information leads to the proposal of a detailed mechanism describing the collision-induced dissociation of duplex DNA (Figure 6). The duplex fragments into single strands by a multistep (preferentially terminal) unzipping. In the course of unzipping, bases can be lost from the duplex. This can in turn induce the backbone fragmentation of one of the single strands to produce Type II fragments, a process that is favored by slow heating conditions. The (DS-B) species can also carry on unzipping to produce the single

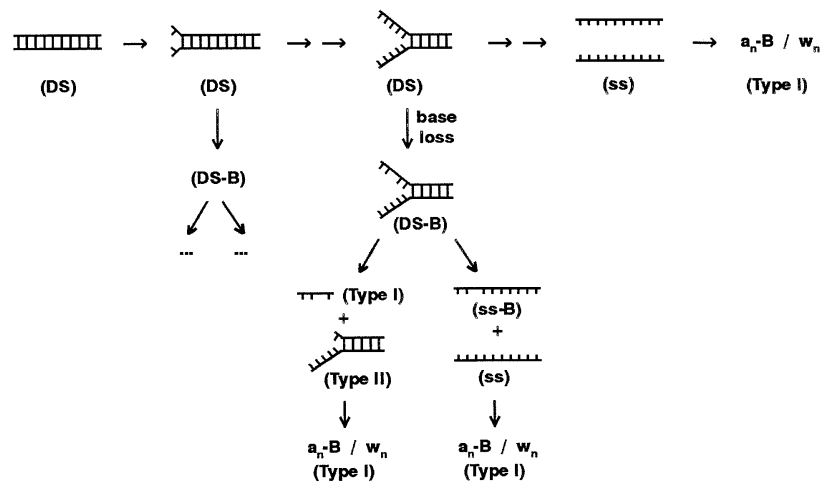

Figure 6. Proposed dissociation mechanism for complementary duplex DNA. Each partially unzipped state could possibly undergo base loss and subsequent fragementation steps that are described in details for one of the species. Base loss can occur anywhere on the partially unzipped part of the single strands (the base loss site on the Figure has been chosen arbitrarily for illustration)

strands, of which one has a missing base. Slow heating conditions extend the time spent in partially unzipped states and thereby favor the neutral base loss and the formation of Type II fragments. Type I fragments can be produced by two different reaction routes: (1) fragmentation of DS-B into Type II and the complementary Type I fragments and (2) fragmentation of the single strands that contain excess energy. The former route is favored by slow heating conditions, while the latter is favored by fast heating conditions. Type I fragments observed for Duplex C in fast heating conditions (Figure 3a) are likely to be issued from this second reaction route: The energy necessary to induce the noncovalent dissociation into single strands is so high that the excess energy in the products is sufficient to cause the backbone fragmentation of the single strands. The case of Duplex C illustrates that for very strong complexes, it can be very difficult to find suitable experimental conditions to observe only the noncovalent dissociation channel.

\section{Reexamination of MS/MS Results on Oligonucleotide Complexes with Drugs}

Minor groove binders. This dissociation mechanism and the understanding of the effect of the collision regime on the appearance of the spectra allows to shed a new light on the interpretation of MS/MS of DNA complexes with noncovalently bound drugs. For minor groove binder complexes with Duplex B (or of similar length and strength), very different fragmentation channels have been reported. Wan et al. [30] performed MS/MS on an LCQ instrument with the standard activation time of $30 \mathrm{~ms}$ and reported the predominant covalent fragmentation of the complex (neutral base loss and formation of Type II fragments) and the minor noncovalent dissociation channel (into one nude single strand and one drug bound single strand). Gabelica et al. [35] performed MS/MS on a Q-TOF instrument and 


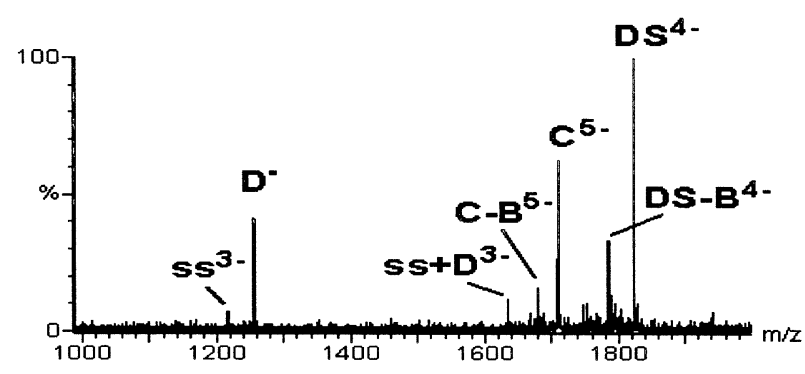

Figure 7. MS/MS spectrum of the complex between Duplex B and actinomycin D, recorded on the Q-TOF2 with a collision energy of $30 \mathrm{eV}$. ( $\mathrm{C}=$ complex, $\mathrm{D}=\mathrm{drug}, \mathrm{DS}=$ double-stranded DNA, ss = single-stranded DNA).

reported the predominant noncovalent dissociation of the complex into the single strands or the single strands bound with a drug (depending on the drug). These apparently contradictory results can now be interpreted by a unique mechanism. Minor groove binders bridge the duplex by forming hydrogen bonds with both strands, and the threshold energy for noncovalent dissociation is higher than for the duplex. Slow heating conditions induce the terminal unzipping of the duplex (the drug is bound to the central AT sequence), the threshold for neutral base loss is reached first and covalent fragmentation occurs. In fast heating conditions, the entropy-favored noncovalent dissociation channel has a larger rate constant, and is indeed observed.

Intercalators. For complexes with intercalators, Wan et al. [30] (MS/MS on LCQ) reported the predominant noncovalent dissociation of the complex. We performed the same MS/MS experiment on the Q-TOF instrument (Figure 7). The spectrum is very similar to that obtained by Wan et al. and we have chosen the same annotations for the ease of comparison ( $\mathrm{C}$ for the complex and $\mathrm{D}$ for the drug), but our interpretation of the peak at 1823.0 Th differs. Although the resolution is not high enough to determine the charge state, we attribute this peak to the $\mathrm{DS}^{4-}$ rather than to the $\mathrm{ss}^{2-}$ because of the observed peak at $1785 \mathrm{Th}$, which is attributed to (DS-B) ${ }^{4-}$. The main fragmentation pathway is the noncovalent dissociation of the complex into the duplex and the negatively charged drug. The duplex therefore keeps only four charges. For complexes with intercalators, the noncovalent dissociation is the channel with the lowest threshold, and is favored at all collision regimes.

\section{Conclusions}

The present article highlights some specific features of the dissociation of duplex DNA that are related to the problem of collision-induced dissociation of noncovalent complexes in general. First, the choice of the experimental conditions (and of the instrument) can have a great influence on the observed dissociation channels of noncovalent complexes when some low- energy (enthalpy-driven) covalent bond cleavages can compete with the noncovalent dissociation channel. As noncovalent dissociation is an entropy-driven process, its observation can be favored by high energy, fast activation conditions. This work also evidences that for complexes containing a high number of contributing interactions organized at the interface of the ligands, the noncovalent dissociation is likely to be a multistep process. This has important implications for the interpretation of the dissociation kinetics. The overall (observed) rate constant does not reflect a one-step dissociation with a single activation energy. Rather, the rate constant is the resultant of consecutive small dissociation steps with little energy barriers. For the observed dissociation rate to reflect the contribution of all noncovalent interactions, there must be no rate-limiting step in the dissociation process.

\section{Acknowledgments}

This work has been supported by the Actions de Recherche Concertées (ARC) (Direction de la Recherche Scientifique-Communauté Française de Belgique). VG is indebted to the F.N.R.S. for a research fellowship.

\section{References}

1. Yamashita, M.; Fenn, J. B. Electrospray Ion Source: Another Variation on the Free-Jet Theme. J. Phys. Chem. 1984, 88, 4451-4459.

2. Whitehouse, M.; Dreyer, R. N.; Yamashita, M.; Fenn, J. B. Electrospray Interface for Liquid Chromatographs and Mass Spectrometers. Anal. Chem. 1985, 57, 675-679.

3. Fenn, J. B.; Mann, M.; Meng, C. K.; Wong, S. F.; Whitehouse, C. M. Electrospray Ionization for Mass Spectrometry. Science 1989, 246, 64-71.

4. Fenn, J. B.; Mann, M.; Meng, C. K.; Wong, S. F. Electrospray Ionization: Principles and Practice. Mass Spectrom. Rev. 1990, 9, 37-70.

5. Thomson, B. A. Declustering and Fragmentation of Protein Ions from an Electrospray Source. J. Am. Soc. Mass Spectrom. 1997, 8, 1053-1058.

6. Chowdhury, S. K.; Katta, V.; Chait, B. T. An Electrospray Ionization Mass Spectrometer with New Features. Rapid Commun. Mass Spectrom. 1990, 4, 81-87.

7. Li, Y.-T.; Hsieh, Y. L.; Henion, J. D.; Ocain, T. D.; Schiehser, G. A.; Ganem, B. Analysis of the Energetics of Gas-phase Immunophilin-ligand Complexes by Ionspray Mass Spectrometry. J. Am. Chem. Soc. 1994, 116, 7487-7493.

8. Rostom, A. A.; Robinson, C. V. Detection of the Intact GroEL Chaperonin Assembly by Mass Spectrometry. J. Am. Chem. Soc. 1999, 121, 4718-4719.

9. Collette, C.; Dehareng, D.; De Pauw, E.; Dive, G. Qualitative Comparison Between the Quantum Calculations and Electrospray Mass Spectra of Complexes of Polyammonium Macrotricyclic Ligands with Dicarboxylic Acids. J. Am. Soc. Mass Spectrom. 2001, 12, 304-316.

10. Van der Kerk-van Hoof, A.; Heck, A. J. R. Covalent and Noncovalent Dissociations of Gas-Phase Complexes of Avoparcin and Bacterial Receptor Mimicking Precursor Peptides Studied by CAD Mass Spectrometry. J. Mass Spectrom. 1999, 34, 813-819.

11. Kempen, E. C.; Colorado, A.; Brodbelt, J. S. Gas-Phase Dissociation Energies of Hydrogen-Bonded Complexes in a Quad- 
rupole Ion Trap Mass Spectrometer. In New Methods for the Study of Biomolecular Complexes; Ens, W; Standing, K. G.; Chernushevich, I. E., Eds.; Kluwer Academic Publishers: Dordrecht, 1998; pp 141-147.

12. Wu, Q.; Gao, J.; Joseph-McCarthy, D.; Sigal, G. B.; Bruce, J. E.; Whitesides, G. M.; Smith, R. D. Carbonic Anhydrase-Inhibitor Binding: from Solution to Gas Phase. J. Am. Chem. Soc. 1997, $119,1157-1158$.

13. Gao, J.; Wu, Q.; Carbeck, J. D.; Lei, Q. P.; Smith, R. D.; Whitesides, G. M. Probing the Energetics of Dissociation of Carbonic Anhydrase-Ligand Complexes in the Gas Phase. Biophys. J. 1999, 76, 3253-3260.

14. Schnier, P. D.; Klassen, J. S.; Strittmatter, E. F.; Williams, E. R. Activation Energies for Dissociation of Double Strand Oligonucleotide Anions: Evidence for Watson-Crick Base Pairing in vacuo. J. Am. Chem. Soc. 1998, 120, 9605-9613.

15. Gross, D. S.; Zhao, Y.; Williams, E. R. Dissociation of HemeGlobin Complexes by BIRD: Molecular Specificity in the Gas Phase? J. Am. Soc. Mass Spectrom. 1997, 8, 519-524.

16. Penn, S. G.; He, F.; Green, M. K.; Lebrilla, C. B. The Use of Heated Capillary Dissociation and Collision-Induced Dissociation to Determine the Strength of Noncovalent Bonding Interactions in Gas-phase Peptide-Cyclodextrin Complexes. J. Am. Soc. Mass Spectrom. 1997, 8, 244-252.

17. He, F.; Ramirez, J.; Garcia, B. A.; Lebrilla, C. B. Differentially Heated Capillary for Thermal Dissociation of Noncovalently Bound Complexes Produced by Electrospray Ionization. Int. J. Mass Spectrom. 1999, 182/183, 261-273.

18. El-Kabbali, O.; Rogniaux, H.; Barth, P.; Chung, R. P. T.; Fletcher, E. V.; Van Dorsselaer, A.; Podjarny, A. Aldose and Aldehyde Reductases: Correlation of Molecular Modeling and Mass Spectrometric Studies on the Binding of Inhibitors to the Active Site. Proteins 2000, 41, 407-414.

19. Hunter, C. L.; Mauk, A. G.; Douglas, D. J. Dissociation of Heme from Myoglobin and Cytochrome b5: Comparison of Behavior in Solution and in the Gas Phase. Biochemistry 2000, 36, 1018-1025.

20. Gabelica, V.; De Pauw, E. Comparison Between Solutionphase Stability and Gas-phase Kinetic Stability of Oligodeoxynucleotide Duplexes. J. Mass Spectrom. 2001, 36, 397-402.

21. Vékey, K. Internal Energy Effects in Mass Spectrometry. J. Mass Spectrom. 1996, 31, 445-463.

22. Forst, W. The Theory of Unimolecular Reactions. Academic Press: New York, 1973, pp 3-142.
23. Gilbert, R. G.; Smith, S. C. Theory of Unimolecular and Recombination Reactions. Blackwell Scientific Publications: Oxford, 1990, pp 52-211.

24. Lorquet, J. C. Whither the Statistical Theory of Mass Spectra. Mass Spectrom. Rev. 1994, 13, 233-257.

25. Baer, T.; Mayer, P. M. Statistical Rice Ramsperger Kassel Marcus Quasiequilibrium Theory Calculations in Mass Spectrometry. J. Am. Soc. Mass Spectrom. 1997, 8, 103-115.

26. Hase, W. L. Some Recent Advances and Remaining Questions Regarding Unimolecular Rate Theory. Acc. Chem. Res. 1998, 31, 659-665.

27. Price, W. D.; Schnier, P. D.; Jockusch, R. A.; Strittmatter, E. F.; Williams, E. R. Unimolecular Reaction Kinetics in the HighPressure Limit Without Collisions. J. Am. Chem. Soc. 1996, 118, 10640-10644

28. Dunbar, R. C.; McMahon, T. B. Activation of Unimolecular Reactions by Ambient Blackbody Radiation. Science 1998, 279, 194-197.

29. Ding, J.; Anderegg, R. J. Specific and Non-Specific Dimer Formation in the Electrospray Ionization Mass Spectrometry of Oligonucleotides. J. Am. Soc. Mass Spectrom. 1995, 6, 159164.

30. Wan, K. X.; Shibue, T.; Gross, M. L. Gas-Phase Stability of Double-Stranded Oligodeoxynucleotide and their Noncovalent Complexes with DNA-Binding Drugs is Revealed by Collisional Activation in an Ion Trap. J. Am. Soc. Mass Spectrom. 2000, 11, 450-457.

31. Penn, S. G.; He, F.; Lebrilla, C. B. Peptides Complexed to Cyclodextrins Fragment Rather than Dissociate when Subjected to Blackbody Infrared Radiation. J. Phys. Chem. B 1998, 102, 9119-9126.

32. McLuckey, S. A.; Vaidyanathan, G.; Habibi-Goudarzi, S. Charged versus Neutral Nucleobase Loss from Multiply Charged Oligonucleotide Anions. J. Mass Spectrom. 1995, 30, 1222-1229.

33. Nordhoff, E.; Kirpekar, F.; Roepstorff, P. Mass Spectrometry of Nucleic Acids. Mass Spectrom. Rev. 1996, 15, 67-138.

34. Klassen, J. S.; Schnier, P. D.; Williams, E. R. Blackbody Infrared Radiative Dissociation of Oligonucleotide Anions. J. Am. Soc. Mass Spectrom. 1998, 9, 1117-1124.

35. Gabelica, V.; De Pauw, E.; Rosu, F. Interaction Between Antitumor Drugs and Double-Stranded DNA Studied by Electrospray Ionization Mass Spectrometry. J. Mass Spectrom. 1999, 32, 1328-1337. 